\title{
Physical Therapy Japan Vol.47 (2020) ABSTRACTS
}

The Japanese Physical Therapy Association publishes the "Journal of the Japanese Physical Therapy Association", the most recent Japanese volume (Physical Therapy Japan) being number 47, which includes 66 articles. Each year 6 issues are published.

For the past 22 years, the English volume of “The Journal of the Japanese Physical Therapy Association” has been published once a year. The journal has changed its' name to "Physical Therapy Research" and the publishing organization changed to Japanese Society of Physical Therapy in 2016, and has published two times per year from 2017, and three times per year from 2021.

To further acquaint our English volume readers with the articles published in the Japanese volume, the English abstracts of the present Japanese volume will be included in the English volume published this year.

\section{Vol. 47, No. 1}

\section{Three-Dimensional Kinematics of the Radiocarpal and Midcarpal Joints of the Wrist during Reverse Dart Throw Motion Using Computed Tomography: To Evaluate the Range and Direction of Motion}

\author{
Kenji KASUBUCHI, PT, MS, Hisao MORITOMO, MD, PhD, Masao YUKIOKA, MD, PhD \\ Department of Physical Therapy, Faculty of Health Science, Osaka Yukioka College of Health Science
}

Kenji KASUBUCHI, PT, MS, Kazuomi SUGAMOTO, MD, PhD

Department of Orthopedic Biomaterial Science, Osaka University Graduate School of Medicine

Hisao MORITOMO, MD, PhD, Sayuri ARIMITSU, MD, PhD, Masao YUKIOKA, MD, PhD

Department of Orthopaedic Surgery, Yukioka Hospital

Purpose: This study aimed to analyze three-dimensional kinematics of the radiocarpal and midcarpal joints of the wrist during reverse dart throw motion.

Methods: We analyzed the kinematics of the radiocarpal and midcarpal joints of the wrists of 12 healthy volunteers using CT images. We measured the range and direction of motion for the radiocarpal and midcarpal joints. Paired t-tests were used to compare the results between the radiocarpal and midcarpal joints.

Results: The range of motion of the radiocarpal joint was significantly greater than that of the midcarpal joint. The motion of the radiocarpal joint in the flexion/extension plane was also significantly greater than that of the midcarpal joint. For the midcarpal joint, the motions of the lateral and central articulations were significantly greater in the radial/ulnar deviation plane compared to those of the radiocarpal joint.

Conclusion: The range of motion of the radiocarpal joint was greater than that of the midcarpal joint during reverse dart throw motion. The radiocarpal joint, especially, contributed to the motion in the flexion-extension plane. The midcarpal joint contributed to the motion in the radial/ulnar deviation plane.

Key Words: Reverse dart throw motion, Three-dimensional kinematics, Radiocarpal joint, Midcarpal joint 


\title{
Effect of Neuromuscular Electrical Stimulation in Postoperative Patients with Femoral Proximal Fractures: A Stratified, Randomized Controlled Trial using a Surgical Approach
}

\author{
Mitsunori TOKUDA, PT, PhD, Daisuke BAI, PT, MSc, Yuki FUJIMORI, PT, Yuka YAMADA, PT, \\ Shingo SUGIMORI, PT, Hiroyuki OKUDA, PT, Taiki IKEMOTO, PT, Yuki MORIKAWA, PT \\ Department of Rehabilitation, Heisei Memorial Hospital \\ Mitsunori TOKUDA, PT, PhD, Koji SHOMOTO, PT, PhD \\ Graduate School of Health Sciences Kio University \\ Daisuke BAI, PT, MSc \\ Graduate School of Medicine, Musculoskeletal Reconstructive Surgery, Nara Medical University
}

Purpose: This study aimed to examine the effect of neuromuscular electrical stimulation (NMES) on leg function and movement ability for muscle strength training of the quadriceps in postoperative patients with femoral proximal fractures.

Methods: Our study was a stratified, randomized controlled trial using a surgical approach in patients with proximal femoral fractures. Eighty-two patients were randomly divided into two groups: the NMES group or control (muscle strength training with no NMES) group. NMES was applied once a day from the next day following surgery. We measured knee extension muscle strength, the Japanese Orthopedic Association (JOA) scores, and the number of days taken for gait and activities of daily living (ADL) to become independent.

Results: Knee extension muscle strength and JOA scores were significantly better in the NMES group. The number of days taken for gait and ADL to become independent were significantly lower in the NMES group. In addition, patients in the NMES group were able to obtain a higher gait ability at discharge than those in the control group.

Conclusion: Training of the quadriceps using NMES from the next day following surgery in postoperative patients with femoral proximal fractures contributed to early improvement in knee extension muscle strength, early acquisition of gait and ADL independence, and improved gait ability at discharge.

Key Words: Femoral proximal fractures, Neuromuscular electrical stimulation: NMES, Gait, Early after surgery, Surgical approach 


\title{
Effects of Simulated Postural Kyphosis on Pulmonary Function and Voluntary Cough Strength
}

\author{
Hiromichi TAKEDA, PT, MS \\ Rihastage Co., Ltd. \\ Hiromichi TAKEDA, PT, MS, Kazuyuki TABIRA, PT, PhD \\ Graduate School of Health Science, Kio University \\ Yoshihiro YAMASHINA, PT, PhD \\ Department of Physical Therapy, Aino University
}

Purpose: The purpose of this study was to investigate the influence of postural kyphosis on voluntary cough strength.

Methods: Sixteen healthy males participated in this study. Cough peak flow, respiratory function, respiratory muscle strength, chest expansion, maximum phonation time, and respiratory impedance indices (respiratory system resistance at $5 \mathrm{~Hz}$, R5; resonant frequency, Fres) were evaluated under four conditions: non-kyphosis, mild kyphosis, moderate kyphosis, and severe kyphosis. One-way analysis of variance and multiple comparison tests (using the Bonferroni method) were performed to compare the four conditions. Correlations between the change rate of each measured variable by the degree of kyphosis were analyzed using Spearman's correlation coefficients.

Results: Cough peak flow, vital capacity, chest expansion, and maximum phonation time were significantly decreased in moderate kyphosis and severe kyphosis compared with those in non-kyphosis. Respiratory muscle strength and Fres were significantly decreased in severe kyphosis compared with those in non-kyphosis. Moreover, there were significant positive correlations between cough peak flow and vital capacity, expiratory muscle strength, and chest expansion at the xiphoid process $(\mathrm{r}=0.27,0.33$, 0.37 , respectively, $\mathrm{p}<0.05)$.

Conclusions: Our data suggest that cough peak flow is reduced due to decreased lower chest expansion, respiratory muscle strength, and vital capacity at moderate or higher kyphosis.

Key Words: Postural kyphosis, Respiratory function, Voluntary cough strength 


\section{Influence of Acute Arm-cranking Exercise with Electrical Muscle Stimulation on Vascular Endothelial Function}

Mizuki ISHIKAWA, PT, MS, Ayako AZUMA, MS, Yasuaki TAMURA, PT, MS,

Akihiko MATSUMOTO, PT

Graduate School of Integrated Arts and Science, Tokushima University

Mizuki ISHIKAWA, PT, MS

Department of Rehabilitation Medicine, Nakazu-yagi Hospital

Hajime MIURA, PhD

Faculty of Integrated Arts and Science, Tokushima University

Purpose: The purpose of this study was to assess the effect of submaximal arm-cranking exercise with EMS on vascular endothelial function.

Methods: Ten healthy young men performed submaximal arm-cranking exercise at $50 \% \mathrm{VO}_{2}$ max for 20 min. All subjects performed submaximal arm-cranking exercise alone (A) and with EMS (A+E). In the $\mathrm{A}+\mathrm{E}$ trial, the submaximal arm-cranking exercise was performed at $50 \% \mathrm{VO}_{2}$ max for 20 min while EMS was applied to their thigh and calf muscles during the exercise. The flow-mediated dilation (FMD) at the brachial artery was measured before and after exercise to calculate the normalized FMD (nFMD).

Results: In the A+E trial, the $\mathrm{nFMD}$ was significantly increased 30 minutes after exercise compared to the rest value and was increased 30 minutes after exercise compared to the A trial.

Conclusion: These findings suggest that arm-cranking exercise with EMS increases vascular endothelial function.

Key Words: Arm-cranking exercise, Electrical muscle stimulation, Brachial artery, Flow-mediated dilation, Vascular endothelial function 


\title{
Study of Responsiveness and Minimal Clinically Important Difference (MCID) of Functional Assessment for Control of Trunk (FACT) in Acute Stroke Patients
}

\author{
Hiroki SUGA, PT, Hironobu KAKIMA, PT, Shota HAYASHI, PT, MSc, Tatsuya IGARASHI, PT \\ Numata Neurosurgery \& Heart Disease Hospital \\ Chiaki SIMA, PT \\ Agatsuma Neurosurgery \& Heart Disease Clinic \\ Kazuhiro MIYATA, PT, PhD \\ Ibaraki Prefectural University of Health Sciences
}

Purpose: This study aimed to examine responsiveness and Minimal Clinically Important Difference (MCID) of Functional Assessment for Control of Trunk (FACT) in patients with acute stroke.

Methods: This study included 30 patients who were hospitalized with stroke. Trunk function was assessed on the 7th day of hospitalization and the final intervention date of physical therapy. For each test, responsiveness and MCID were examined. Improvement in activity of daily living was used to detect a clinically meaningful change. The MCID for the trunk function assessment tool was estimated by meaningful change.

Results: FACT, a tool used to assess trunk function, showed a large degree of responsiveness. The MCID of FACT was 4 points, and its discrimination accuracy was very high.

Conclusions: This study indicated FACT had good responsiveness and high accuracy in discrimination of MCID. These results suggest that FACT is useful trunk function assessment tool in patients with acute stroke.

Key Words: Stroke, Trunk function assessment tool, FACT, Responsiveness, MCID 
Factors Associated with Re-Admission with Nursing and Healthcare-Associated Pneumonia

Hiromi FUKUDA, PT, Muneyoshi NISHIDA, PT, Yusuke ISHIBASHI, PT, Takahiro KANDA, PT

Department of Physical Therapy, Akitsukounoike Hospital

Hisae HAYASHI, PT, PhD

Department of Rehabilitation and Care, University of Seijoh

Purpose: The purpose of this study is to determine factors affecting re-admission with nursing and healthcare-associated pneumonia (NHCAP). Design: Single-center, retrospective clinical study.

Method: A total of 123 patients who readmitted to the hospital due to NHCAP from January 2016 to November 2016 were reviewed. Clinical characteristics, laboratory test values, Functional Independence Measure score (FIM score), the status of nursing care insurance services, social information, and methods of providing information were extracted from medical records. Cox proportional hazards analysis was performed to determine the factors affecting readmission.

Results: Age, FIM score (exercise items) at discharge, serum albumin, the days between admission and first ambulation, face-to-face communication were detected as significant factors.

Conclusion: This study provides valuable information about factors leading to readmission with NHCAP.

Key Words: Nursing and healthcare-associated pneumonia, Re-admission, Hospitals for Communitybased Care 
The Relationship between the Step Side Knee Joint Movement and Elbow Valgus Torque

Tomoya UCHIDA, PT, MS, Hiroyuki FURUKAWA, PT, PhD, Shintaro MATSUMOTO, PT,

Minoru KOMATSU, PT, MS, Michiru TSUKUDA, PT, Hiroyuki DONJO, PT,

Kenji FUJITA, MD

Fujita Orthopaedic \& Sports Clinic

Satoshi OKUBO, PT, MS

Kobegakuin University

Purpose: The purpose of this study was to investigate that the characteristic of the step leg motion that increase elbow valgus torque.

Method: Twenty-seven youth baseball pitchers participated in this study. Elbow valgus torque measurement and video analysis were performed on outdoor mound. The participants divided by the qualitative assessment of stride knee motion, then compared elbow valgus torque and flexion angle of step knee joint between both groups.

Result: There was significant greater flexion knee joint angle at maximum external rotation and ball release and greater joint torque of elbow and less ball speed for the forward movement group.

Conclusion: This group showed inadequate bracing the knee of step leg. It caused increasing elbow torque. To evaluate the stride leg motion is important for assessment of risk on elbow injury.

Key Words: Pitching motion, Elbow values torque, The motion of the step leg 


\title{
Examination of the Factor Related to the Acute Phase Motor Functional Prognosis of Branch Atheromatous Disease
}

\author{
Kazuhiro TOKUDA, PT, Kazuya KAISE, PT \\ Department of Rehabilitation, Hanwa Memorial Hospital \\ Takashi TAKEBAYASHI, OT, PhD \\ Department of Occupational Therapy, School of Comprehensive Rehabilitation, Osaka Prefecture University \\ Takashi KOYAMA, MD, Toshiaki FUJITA, MD \\ Department of Cranial Nerve Surgery, Hanwa Memorial Hospital
}

Purpose: The acute motor functional prognosis was evaluated in patients with branch atheromatous disease (BAD).

Method: Overall, 101 patients with BAD were enrolled from March 2016 to February 2019. Patients with paralysis in their upper limbs and lower limbs were classified into good and bad groups based on their paralysis status at discharge. The patients' age, gender, paralyzed side, National Institute of Health Stroke Scale (NIHSS) at admission, lesion area, infarct area, rehabilitation start date, Fugl-Meyer Assessments (FMA) of the upper and lower limbs, starting Mini-mental State Examination (MMSE) score, hospitalization days, OT session, and PT session were analyzed. Thereafter, univariate and logistic regression analyses were performed.

Results: The univariate analysis revealed significant differences in age, NIHSS at admission, infarct area, FMA, MMSE at initiation, and hospital days in both patients with paralysis in their upper and lower limbs. The logistic regression analysis revealed that FMA was an independent factor in both patients with paralysis in their upper and lower limbs. The cut-off values were 18 for the upper limb (area under the curve, 0.94; sensitivity, 0.80; and specificity, 0.93) and 19 for the lower limb (area under the curve, 0.88; sensitivity, 0.80 ; and specificity, 0.82 ).

Conclusion: The initial FMA in patients with BAD was most relevant to motor functional prognosis from the acute phase in the upper and lower limbs.

Key Words: Stroke, Acute phase, Prognosis prediction, Motor paralysis 
Characteristics of Sagittal Knee Joint Kinematics Dynamics and Quadriceps Muscle Activity during Stair Ascent and Descent in Patients after Total Knee Arthroplasty

Taiki FURUMOTO, PT, Tsuyoshi GOTO, PT, MSc, Shinsuke KATOH, MD, PhD

Department of Rehabilitation, Tokushima University Hospital

Taiki FURUMOTO, PT, Daisuke HAMADA, MD, PhD, Tsuyoshi GOTO, PT, MSc,

Tomoya TAKASAGO, MD, Koichi SAIRYO, MD, PhD

Department of Orthopedics, Institute of Biomedical Sciences Tokushima University Graduate School

Purpose: The aim of this study was to evaluate the characteristics of sagittal knee joint kinematics and quadricep muscle activity during stair climbing in patients after total knee arthroplasty (TKA).

Methods: Eight and ten limbs were included in the TKA and control groups, respectively. The maximum knee extension moment (KEM) and maximum afferent and centrifugal knee power during stair climbing stance phases were measured by a three-dimensional motion analysis system. Maximum muscular activity of the quadriceps femoris was measured by surface electromyography.

Results: The knee joint load and afferent power in stair ascent of the TKA group was significantly lower than that of the control group. The centrifugal power in stair descent of the TKA group was significantly lower than that of the control group. Quadriceps activity during stair ascent was significantly higher in the TKA group than in the control group, and the descending muscle activity was low in both groups.

Conclusion: One year post-operation, TKA patients strongly contract the quadriceps during stair climbing, but the joint load and afferent power decreased during ascension and the centrifugal power decreased during descension.

Key Words: Total knee arthroplasty, Stair climbing, Sagittal knee joint kinematics dynamics, Quadriceps femoris muscle activity 


\section{Effectiveness of Electrostimulation of the Quadriceps for 4 days in the Acute Phase after Total Hip Arthroplasty}

Osamu WADA, PT, MSc, Tomohiro OKA, PT, MSc

Anshin-Hospital

Yousuke YAMAMOTO, PT

Anshin-Clinic

Purpose: This study aimed to investigate the effectiveness of electrostimulation of the quadriceps for 4 days in the acute phase after total hip arthroplasty (THA).

Methods: In total, 52 women with hip osteoarthritis participated in this study. They were randomized to the control and intervention groups. The intervention group $(n=26$; age $64.4 \pm 8.9$ years) underwent electrostimulation of the quadriceps twice a day during knee extension exercises for 4 days. The control group ( $\mathrm{n}=26$; age $66.0 \pm 9.9$ years) performed knee extension exercises twice a day without electric muscle stimulation for 4 days. Additionally, both groups received the standard protocol in our hospital. Quadriceps strength, gait speed, and pain during gait in both groups were assessed 1 month before surgery and on Day 4 after surgery. Changes in quadriceps strength and gait speed were quantified as relative (percent) changes from the preoperative values ( $\Delta$ quadriceps strength and $\Delta$ gait speed). Changes in pain during gait were quantified as relative (difference) changes from the preoperative values $(\Delta$ pain during gait). The Mann-Whitney $U$ test and unpaired t-test were used to compare these values between the groups. Significance was set at $\mathrm{P}<0.05$.

Results: Compared with the control group, the intervention group showed greater $\Delta$ quadriceps strength $(\mathrm{P}=0.014)$, $\Delta$ gait speed $(\mathrm{P}=0.009)$, and $\Delta$ pain during gait $(\mathrm{P}=0.027)$.

Conclusions: Electrostimulation of the quadriceps for 4 days in the acute phase after THA improves knee extensor strength and gait speed, and reduces pain during gait.

Key Words: Total hip arthroplasty, Electrostimulation, Knee extensor strength 


\section{Effect of Glenohumeral External Rotation Position on the Posterior Glenohumeral Distance in the Throwing Shoulders of College Baseball Players: An MRI Study of the Simulated Late Cocking Phase during Baseball Throwing}

Makoto TAKAHASHI, PT, MS

Department of Rehabilitation, Hitachino Orthopedic Clinic

Koji IWAMOTO, PhD, Masafumi MIZUKAMI, PhD

Department of Physical Therapy, Ibaraki Prefectural University of Health Sciences

Masahiko MONMA, PhD

Department of Radiological Sciences, Ibaraki Prefectural University of Health Sciences

Purpose: The purpose of this study was to assess the effects of external glenohumeral rotation position on the posterior glenohumeral distance (PGHD) in asymptomatic throwing shoulders.

Methods: Eleven asymptomatic male college baseball players (11 throwing shoulders) participated in this research. The PGHD was calculated using MRI scans. MRI measurement positions were $90^{\circ}$ of shoulder abduction with external rotations of $90^{\circ}, 100^{\circ}$, and $110^{\circ}$.

Results: Measures of PGHD were significantly less during $110^{\circ}$ external rotation compared to $90^{\circ}$ external rotation.

Conclusion: The PGHD was significantly less when shoulder abduction occurred in more externally rotated positions.

Key Words: Shoulder joint, Glenohumeral external rotation position, Posterior glenohumeral distance 


\section{Association of Back and Knee Pain with Physical Function in Community-dwelling Older Adults: Effects of Acute/Chronic Pain on Gait Speed and Grip Strength}

Yuki NAKAI, PT, MS, Ryoji KIYAMA, PT, PhD, Hyuma MAKIZAKO, PT, PhD

School of Health Sciences, Faculty of Medicine, Kagoshima University

Kazutoshi TOMIOKA, PT, MS, Yoshiaki TANIGUCHI, PT, Nana SATO, PT, Ayumi WADA, PT

Graduate School of Health Sciences, Kagoshima University

Takuro KUBOZONO, MD, PhD, Mitsuru OHISHI, MD, PhD

Graduate School of Medical and Dental Sciences, Kagoshima University

Hyuma MAKIZAKO, PT, PhD

Department of Preventive Gerontology, Center for Gerontology and Social Science, National Center for Geriatrics and Gerontology

Purpose: Approximately $80 \%$ of community-dwelling older adults present with musculoskeletal pain accompanied by low gait speed and grip strength. This study investigated the effects of acute or chronic back and knee pain on gait speed and grip strength in community-dwelling older adults.

Methods: This study included 735 community-dwelling older adults (aged $\geq 65$ years, mean age 74.8 years, 62.9\% women) (Tarumizu study 2018). Type of pain (acute vs. chronic) was determined using a questionnaire. We investigated the association between acute or chronic pain and low gait speed $(<1.0 \mathrm{~m} /$ s) and low grip strength (men $<26 \mathrm{~kg}$, women $<18 \mathrm{~kg}$ ).

Results: Of the 735 participants, 347 (47.2\%) reported no pain, 144 (19.6\%) reported acute pain, and 244 (33.2\%) reported chronic pain. Low back and knee pain were observed in 121 participants (16.5\%), of which $46(38.0 \%)$ reported acute pain and 75 (62.0\%) reported chronic pain. Logistic regression analysis revealed that low gait speed was significantly associated with chronic pain (adjusted odds ratio 2.55, p $=0.001$ ) but not with acute pain (adjusted odds ratio 1.19, $\mathrm{p}=0.632$ ) (adjusted for age, sex, the 15-item Geriatric Depression Scale, medication use, the Appendicular Skeletal Muscle Mass Index, and grip strength). Notably, no association was observed between pain and low grip strength.

Conclusion: Community-dwelling older adults with chronic low back and knee pain showed low gait speed.

Key Words: Community-dwelling older adults, Gait speed, Grip strength, Chronic pain, Acute pain 


\section{Effects of Physical Therapy in the Patients with Degenerative Disorders of the Cervical Spine: Combined Effects of McKenzie Method, Deep Cervical Muscle Exercise, and Physical Agents}

Kiyonori YO, PT, PhD

Department of Rehabilitation, Hamawaki Orthopaedic Clinic

Eiki TSUSHIMA, PT, PhD

Hirosaki University Graduate school of Health Sciences

Masaaki MURASE, MD, PhD, Yosuke OISHI, MD, PhD

Department of Orthopedic Surgery, Hamawaki Orthopaedic Hospital

Purpose: This study investigated the combined effects of the McKenzie method, deep cervical muscle exercise, and physical agents in patients with degenerative disorders of the cervical spine.

Methods: Study participants included 112 patients diagnosed with degenerative disorders of the cervical spine at our hospital. The patients were divided into three therapeutic groups, which were treated with: the McKenzie method and physical agents (MDT group, 51 cases); the McKenzie method, deep cervical muscle exercise, and physical agents (DCME group, 43 cases); and physical agents alone (physical agents group, 18 cases). The effects of these therapies were evaluated using the active ROM of cervical spines (hereafter, CROM), Neck Disability Index (NDI), JOACMEQ, Visual Analogue Scale (VAS) scores for neck pain, and SF-8 before therapy and 1, 2, 3, and 5 months after therapy. Statistical analysis was performed using the mixed-effects model for repeated measures.

Results: The following evaluation items showed interaction. Compared with before therapy, CROM, NDI, JOACMEQ, and SF-8 scores were significantly improved 1 month after therapy in the MDT and DCME groups. VAS scores significantly improved 1 month after therapy in the MDT and DCME groups, and 2 months after therapy in the physical agents group. These effects were highest in the MDT group, followed by the DCME group and the physical agents group.

Conclusion: Compared to physical agents alone, MDT and DCME resulted in greater symptomatic, functional, and psychological improvement.

Key Words: Degenerative disorders of the cervical spine, McKenzie method, Deep cervical muscle exercise, Physical agents 


\section{The Effect of Nutritional Status on Walking Ability at Discharge in Patients with Acute Exacerbations of COPD}

Takayuki KOBAYASHI, PT, Shinya MATSUSHIMA, PT, MSc, Shun AIKAWA, PT

Department of Rehabilitation Medicine, St. Marianna University School of Medicine Yokohama City Seibu Hospital

Hitoshi YOKOYAMA, PT, MSc, Rie TAKEICHI, PT, Yosuke WATANABE, PT, MSc, Atsushi NAKAKUKI, PT

Center of Rehabilitation Medicine, St. Marianna University School of Medicine Hospital

Shuichi NAKADA, PT

Department of Rehabilitation Medicine, Kawasaki Municipal Tama Hospital

Yuko KOMASE, MD

Department of Respiratory Medicine, St. Marianna University School of Medicine Yokohama City Seibu Hospital

Masamichi MINESHITA, MD

Department of Respiratory Medicine, St. Marianna University School of Medicine Hospital

Purpose: This retrospective study aimed to clarify the associations between nutritional status and walking ability at discharge in patients with acute exacerbation of chronic obstructive pulmonary disease (AECOPD).

Methods: We enrolled 101 patients hospitalized for AECOPD and who underwent physical therapy. Walking ability was assessed by Functional Independence Measure (FIM). The subjects were classified into the independent group (FIM $\geq 6$ ) and non-independent group (FIM $<6$ ) by walking ability at discharge. The presence of malnutrition (\% ideal body weight $<80 \%$ ) and adequacy of caloric intake (\% estimated target calories: intake/estimated target calories $\times 100$ ) were examined as indices of nutritional status. Multivariate logistic regression analysis was performed to assess whether nutritional status affected walking ability at discharge (FIM $\geq 6$ or FIM <6) adjusted by age, A-DROP, and severity of airflow obstruction.

Results: Despite being independent before hospitalization, 21 patients could not walk independently at discharge, and 31 patients had malnutrition before admission. The average \% estimated target calories was $78.7 \pm 31.7 \%$. Multiple logistic regression analysis showed presence of malnutrition (OR 3.9, 95\% CI 1.3-11.7, $\mathrm{p}<0.05$ ) and \% estimated target calories (OR $0.7,95 \%$ CI $0.5-0.9, \mathrm{p}<0.05$ ) to be significantly associated with walking ability at discharge.

Conclusion: Nutritional status affected walking ability of AECOPD patients at discharge. Therefore, nutritional status should be evaluated when providing effective exercise and adequate nutritional therapy during hospitalization. These findings may imply that oral nutritional supplements should be provided immediately after hospitalization for patients with inadequate caloric intake.

Key Words: Acute exacerbation of COPD, Walking ability, Nutritional status 


\section{Feasibility of an Exercise Therapy in Lymphoma Patients with Cytopenia: Preliminary Study by Retrospective Observational Study}

Ryuichi KASAHARA, PT, Shoko TAKAHASHI, PT, Ryohei JINBO, PT, Kazumi JINBO, PT,

Aya TAKANO, PT, Yuichi YAMAMOTO, PT

Kita-Fukushima Medical Center, Department of Rehabilitation

Ryuichi KASAHARA, PT, Takayuki IKEZOE, MD, PhD

Fukushima Medical University, Department of Hematology

Takaaki FUJITA, OT, PhD

Tohoku Fukushi University, Faculty of Health Sciences, Department of Rehabilitation

Tatsuyuki KAI, MD, PhD, Hideo KIMURA, MD, PhD, Yutaka SHIGA, MD, PhD,

Miki FURUKAWA, MD, PhD

Kita-Fukushima Medical Center, Department of Hematology

Shinichiro MORISHITA, PT, PhD

Niigata University of Health and Welfare, Department of Physical Therapy

Purpose: This study aims to assess the relationship between blood data and the safety and feasibility of physical therapy in lymphoma patients with cytopenia.

Methods: Total 79 patients with lymphoma who underwent chemotherapy were included in this study. All patients received exercise therapy (13 on the Borg scale) six days per week, 20 min per day. Physical therapy adherence (implementation/scheduled days) was calculated after stratification according to the white blood cell and platelet counts. In addition, the occurrence of adverse events was investigated.

Result: The median physical therapy adherence rate of the patients was high at $96.8 \%$ (range, $61.7 \%$ 100.0\%), and no adverse events were observed during or after the physical therapy. However, the adherence rate significantly decreased when the white blood cell count was $<1,000 / \mu \mathrm{L}$ and platelet count was $<20,000 / \mu \mathrm{L}(\mathrm{p}<0.001)$.

Conclusion: The results of this study suggest the safety of exercise therapy in patients with lymphoma. In addition, the results suggest that decreased white blood cell and platelet counts are associated with poor physical therapy adherence.

Key Words: Cytopenia, Malignant lymphoma, Feasibility 
The Reliability and Validity of the Trunk Impairment Scale for Patients with Spastic Cerebral Palsy

Yasuaki KUSUMOTO, PT, PhD

Tokyo University of Technology

Kanako FUJII, PT, PhD

Seiyo Gakuen

Hiroto HAYASHI, PT

ITO CO., LTD

Kenji TAKAKI, PT, MSc

Department of Physical Therapy, Faculty of Health Sciences, Mejiro University

Satsuki AMIMOTO, PT

General Incorporated Association Para SC Esperanza

Tadamitsu MATSUDA, PT, PhD

Department of Physical Therapy, Faculty of Health Sciences, Juntendo University

Osamu NITTA, PT, PhD

Department of Physical Therapy, Faculty of Health Sciences, Tokyo Metropolitan University

Purpose: This study aimed to assess the reliability and validity of the Japanese version of the Trunk Impairment Scale (TIS-J) for patients with spastic cerebral palsy.

Methods: A cross-sectional study was conducted, in which a total of 69 patients were enrolled. The reliability of the results were tested for 20 patients by intra-rater and inter-rater reliabilities and minimal detectable change at 95\% confidence interval (MDC95), and construct validity by comparing them with the Gross Motor Function Classification System scores.

Results: The intra-class and interclass correlation coefficients for test-retest reliability were 0.90-0.99. The intra-rater MDC95 values for static sitting balance, dynamic sitting balance, coordination, and total score were $0.44,1.35,0.44$, and 0.96 , respectively, while the inter-rater MDC95 values for the same were $1.54,1.97,1.15$, and 2.37. The Spearman correlation coefficient were $-0.63,-0.76,-0.30$, and -0.74 . It revealed a good relationship for validity between the change in score for the dynamic sitting balance and total TIS-J scores.

Conclusions: The TIS-J was found to be reliable and valid for patients with spastic cerebral palsy.

Key Words: Cerebral palsy, Trunk Impairment Scale, Reliability, Validity, Function of the trunk 


\section{Representative Value of Nutritional Status and Physical Function Stratified by Age and Sex in Maintenance Hemodialysis Patients}

Ayaka HARA, PT

Dialysis Division at Shizuoka, Kaishoukai

Kenichi KONO, PT, PhD, Yusuke NISHIDA, PT, PhD

International University of Health and Welfare School of Health Science at Narita Department of Physical Therapy

Hiroyo OSHITA, MD, Humiko NAGASHIMA, NS, Shinya NAMURA, CE, Hiroshi ICHIYANAGI, CE Johoku Kyoritsu Clinic, Kaishoukai

Hiroki YABE, PT, PhD

Seirei Christopher University, School of Rehabilitation Department of Physical Therapy

Yoshihumi MORIYAMA, AT

Department Wellness Center, Kaikoukai Nagoya Kyoritsu Hospital

Tetsuya YAMADA, MD, PhD

Dialysis Division, Kaikoukai Healthcare Group

Purpose: The aim of this study was to clarify the representative values and the proportion below the cut off value related to nutritional status and physical function in maintenance hemodialysis patients.

Methods: A total of 670 hemodialysis outpatients participated in this cross-sectional study. Patient's background, body mass index (BMI), geriatric nutritional risk index (GNRI), grip strength, knee extension force (KEF), gait velocity, and short physical performance battery (SPPB) were investigated. The validity of the sample size was confirmed, and representative values and the proportions below the cut off values were compared.

Results: A sufficient sample size was able to be set for patients aged 50-years and older. GNRI, grip strength, KEF, gait velocity, and SPPB decreased with increasing age, and many patients aged 80 years or older were below the cut-off value.

Conclusion: This study showed the representative values of nutritional status and physical function by age and demonstrated an actual decrease in elderly patients.

Key Words: Hemodialysis, Physical function, Representative value 


\title{
Prediction Accuracy of Walking Independence in Mini-Balance Evaluation Systems Test with Spinocerebellar Degeneration
}

\author{
Keisuke OTA, PT, Kousuke SAKANO, PT, Yuuichi NAKASHIRO, PT \\ Department of Rehabilitation, Hokuyu-kai Neurological Hospital \\ Hiroki MANI, PT, PhD \\ Department of Rehabilitation Science, Faculty of Health sciences, Hokkaido University \\ Fumio MORIWAKA, MD, PhD \\ Department of Neurology, Hokuyu-kai Neurological Hospital
}

Kazuhiro MIYATA, PT, PhD

Department of Physical Therapy, Ibaraki Prefectural University of Health Science

Purpose: We aimed to investigate whether the Mini-Balance Evaluation Test (Mini-BESTest) can be applied to assess walking impairment severity in individuals with spinocerebellar degeneration (SCD) and to compare the difference in walking impairment severity assessed using Mini-BESTest and Berg Balance Scale (BBS).

Methods: Thirty individuals with SCD participated in the study and were divided into three groups according to the SCD severity classification. They were assessed using Mini-BESTest, BBS, and Functional Independence Measure (FIM). The total scores and distribution of scores from Mini-BESTest and BBS were compared among the three groups. In addition, a receiver-operating characteristic (ROC) plot was used to compare the overall accuracy in assessing walking severity using Mini-BESTest and BBS and to determine appropriate cutoff scores for identifying whether an individual can walk independently.

Results: The total Mini-BESTest and BBS scores showed significant differences among the three groups. Both scores decreased with an increase in walking impairment severity. Mini-BESTest and BBS had high area under the curve and sensitivity and specificity. A ceiling effect and deviation in bias in the distribution were identified only on BBS.

Conclusion: Mini-BESTest had a higher accuracy and sensitivity than the BBS was thus considered useful for assessing properties that identify individuals with SCD who can walk independently.

Key Words: Spinocerebellar Degeneration, Balance ability, Mini-BESTest, Walking independence 


\section{Relevance of Scapula Function and Preoperative Factor after \\ Reverse Total Shoulder Arthroplasty}

Takuya MAEDA, PT, Hisayo OZAKI, PT, PhD

Showa University Hospital

Takashi IKEDA, PT, PhD, Hisayo OZAKI, PT, PhD, Shinichi CHIBA, PT, PhD

Showa University, Dept of School of Nursing and Rehabilitation Sciences

Takashi IKEDA, PT, PhD, Masaki TAMURA, PT, MSc, Hisayo OZAKI, PT, PhD,

Naoya NISHINAKA, MD, PhD, Masashi SUZUKI, MD, PhD, Shinichi CHIBA, PT, PhD

Showa University Reseach Institute for Sport and Exercise Sciences

Takashi IKEDA, PT, PhD, Masaki TAMURA, PT, MSc

Showa University Fujigaoka Rehabilitation Hospital

Masaki TAMURA, PT, MSc

Graduate school of Health Science, Showa University

Naoya NISHINAKA, MD, PhD, Masashi SUZUKI, MD, PhD

Department of Orthopaedic Surgery, Showa University Fujigaoka Hospital

Shinichi CHIBA, PT, PhD

Showa University East Hospital

Purpose: To confirm the relevance of preoperative factors based on post-reverse total shoulder arthroplasty (RTSA) scapula function using scapula-45 radiography.

Method: Twenty-eight patients were observed for more than 6 months after RTSA. We evaluated how the preoperative physical findings had an influence on their postoperative scapular index, which indicates postoperative scapula function.

Results: The only significant independent variable that could be extracted for the postoperative scapular index was the preoperative scapular index.

Conclusion: The only factor affecting the postoperative scapular index is the preoperative scapular index.

Key Words: Reverse total shoulder arthroplasty, RSA, RTSA, Scapula index, Scapular notching 


\section{Electromyographic Analysis of Shoulder Muscle Activity after Reverse Shoulder Arthroplasty: A Comparison with Healthy Shoulders}

Tadashi NAKANO, PT, MS

Division of Physical Therapy, Department of Rehabilitation Sciences, Faculty of Allied Health Sciences, Kansai University of Welfare Sciences

Hisayoshi MURANISHI, PT, MS

Department of Physical Therapy, Faculty of Rehabilitation, School of Rehabilitation, Division of Physical Therapy, Osaka Kawasaki Rehabilitation University

Masaki KUWANO, PT, MS

Department of Rehabilitation, Tsuji Geka Rehabilitation Hospital

Teruya YUKAWA, PT, Yuuki UETANI, PT, Meguru DOI, PT

Department of Rehabilitation, Ito Clinic, Osaka Shoulder Center

Yoichi ITO, MD, PhD

Ito Clinic, Osaka Shoulder Center

Department of Orthopedic Surgery Osaka City University Graduate School of Medicine

Purpose: To analyze shoulder muscle activity after reverse shoulder arthroplasty (RSA) using electromyography to identify any differences from the muscle activity of healthy shoulders.

Methods: The subjects included 12 RSA-treated shoulders and 17 healthy (control) shoulders. Shoulder muscle activity was evaluated using surface electromyography at $45^{\circ}$ and $90^{\circ}$ of shoulder elevation. The subjects held their arms aloft in three directions during recording at flexion, scaption, and abduction. Ratios of integrated electromyographic activity for different muscles at $45^{\circ}$ and $90^{\circ}$ were calculated and compared between the two groups.

Results: Muscle activity ratios in the posterior deltoid during flexion, the upper trapezius and posterior deltoid during scaption, and the clavicular pectoralis major during abduction were significantly higher in the RSA group $(\mathrm{p}<0.05)$.

Conclusions: Our results suggest that RSA-treated shoulder muscles behave differently from healthy shoulders during sustained elevation between $45^{\circ}$ and $90^{\circ}$.

Key Words: Reverse total shoulder arthroplasty, Electromyography, Massive rotator cuff tears, Muscle activity 


\title{
Usefulness of Six Minute Walking Distance as a Predictor of Complications after Gastric Cancer Laparotomy
}

\author{
Yuto ZOGO, PT, Kensuke SERIZAWA, OT, Keisuke MIZUMORI, PT, Akira IWASE, OT, \\ Ayano MASUI, PT, Rema ONODERA, PT, Takashi MATSUMOTO, MD, PhD \\ Rehabilitation Center, Yokohama City Minato Red Cross Hospital \\ Hidetaka ONO, MD, PhD \\ Department of Surgery, Yokohama City Minato Red Cross Hospital \\ Junichiro INOUE, PT, PhD \\ Division of Rehabilitation Medicine, Kobe University Hospital
}

Purpose: We investigated the factors significantly associated with postoperative complications after laparotomy for gastric cancer.

Methods: The study included 24 patients who underwent perioperative rehabilitation before surgery. Patients were categorized into the postoperative complication and the non-postoperative complication groups, and intergroup comparison was performed for statistical analysis. Logistic regression analysis was used to identify factors significantly associated with postoperative complications. The cutoff value was calculated using the receiver operating characteristic (ROC) curve.

Result: The preoperative 6-min walk distance was significantly lower in the postoperative complication group $(257.7 \mathrm{~m}$ vs. $353.1 \mathrm{~m}, \mathrm{p}<0.01)$. Logistic regression analysis showed that the selected factor was only the preoperative 6-min walk distance, and the cutoff value based on the ROC curve was $300 \mathrm{~m}$.

Conclusion: We observed that the preoperative 6-min walk distance was significantly associated with postoperative complications after laparotomy performed for gastric cancer. Therefore, the preoperative 6-min walk distance might effectively predict complications after laparotomy for gastric cancer. Active prehabilitation and improvement of preoperative exercise tolerance are important in patients showing a 6-min walk distance $<300 \mathrm{~m}$.

Key Words: Gastric cancer, Postoperative complication, Six minute walking distance, Cutoff value 
Relationship between N-type Test and Kinder Infant Development Scale

\author{
Hirotaka GIMA, PT, PhD \\ Tottori University \\ Mihoko GIMA, PT \\ Tottori Nursing Association \\ Daiki ASANO, PT, MS \\ Japan Baptist Hospital
}

Purpose: This study aimed to examine the relationship between N-type test and each developmental domain of the Kinder Infant Development Scale (KIDS).

Methods: This study included 42 children aged 3-6 years along with their parents. The N-type motor image test for toddlers (N-test) was used to evaluate motor imagery, and scores were calculated for selecting picture cards representing posture (card task) and for change in posture (postural task). Parents answered questions from the KIDS (Type C), and each subscale was scored (etc. physical-motor, receptive language, expressive language, social relationships with children, and social relationships with adults). These scores were then used to examine the relationship between the N-test scores and the development of each domain.

Results: 32 children completed the N-test. The scores of the N-test and age (in months) showed a significantly positive correlation. The scores for the card task were significantly related to the scores for physical-motor, receptive language, and expressive language from the KIDS. The scores for the postural task were significantly related to the age (in months) as well as scores for physical-motor, receptive language, social relationships with children, and social relationships with adults from the KIDS.

Conclusion: Our results suggest that the tasks of the N-test are associated with the development of different domains. Postural tasks are related to social development, and it is hypothesized that interpersonal communication experience may be involved in the function of motor imagery.

Key Words: Motor imagery, Development, Childhood, Sociality 


\title{
Examination of Factors Associated with Fear of Falling in Elderly People Hospitalized Due to Fractures and Discharged to Their Homes
}

\author{
Kazuyuki SATO, PT, MS \\ Department of Rehabilitation, Tokyo Angel Hospital \\ KK, Silver Assist \\ Rika MANGYO, PT, PhD \\ Department of Physical Therapy, Faculty of Health Sciences, Mejiro University \\ Hisashi KAWAI, PhD, Shuichi OBUCHI, PT, PhD \\ Tokyo Metropolitan Geriatric Hospital and Institute of Gerontology
}

Objective: To comprehensively examine factors associated with fear of falling in elderly who were hospitalized due to fractures and discharged to their homes.

Methods: This study included 39 participants aged $>65$ years who sustained a bone fracture injury and were discharged from our hospital. Relationships between fear of falling and the number of falls, motor function, and residential environmental risk were investigated using Spearman or Pearson correlation analyses. A multiple regression analysis was performed with fear of falling as the dependent variable and independent variables determined using results of the correlation analysis.

Results: The factors significantly correlated with the fear of falling were the number of falls, Berg Balance Scale (BBS), Timed Up and Go test, 10-m walk test, Functional Independence Measure, Tokyo Metropolitan Institute of Gerontology index of competence, and residential environmental risk. In the multiple regression analysis, BBS, residential environmental risk, and the number of falls were identified as factors related with the fear of falling.

Conclusion: External factors such as residential environmental risk as well as common internal risks should be evaluated in elderly patients who sustained fracture to reduce fear of falling.

Key Words: Fear of falling, Fracture, Elderly people, Residential environment, Fall prevention 


\title{
Pelvic Floor Muscle, Trunk Muscles, and Lower Limb Muscles Co-contraction at the Positions of Increasing Abdominal Pressure in Young Nulliparous Subjects
}

\author{
Yui TAKAHASHI, PT \\ Department of Rehabilitation, Aizen Hospital
}

Yui TAKAHASHI, PT, Takeya KITTA, MD, PhD, Mifuka OUCHI, PT, MS,

Nobuo SHINOHARA, MD, PhD

Department of Renal and Genitourinary surgery, Graduate School of Medicine, Hokkaido University

Tsuneo KUMAMOTO, PT, PhD, Toshiaki SEKO, PT, MS

Department of Rehabilitation, Faculty of Health Sciences, Hokkaido Chitose College of Rehabilitation

Tsuneo KUMAMOTO, PT, PhD

Department of Physical Therapy, School of Health and Social Services, Saitama Prefectural University

Sayo MIURA, PT

Department of Rehabilitation, Hokusei Hospital

Yumeko KUDOU, PT

Sapporo Maruyama Orthopedic surgery Miyanosawa Orthopedics Clinic

Yui MATSUDA, Ns

Shinsapporo Neurosurgical Hospital

Takahisa NAGAI, MD

Ladies Clinic Kitahama

Purpose: This study aimed to investigate the co-contraction of the pelvic floor muscle (PFM), trunk muscle, and lower limb muscles at different positions when the abdominal pressure is increased.

Methods: The subjects were 15 young nulliparous women, mean age $25.5 \pm 2.5$ years. We measured vaginal pressure at rest and during PFM contraction. Simultaneously, we also measured the rectus abdominis muscle, external oblique muscle, internal oblique muscle (IO), multifidus muscle, gluteus maximus muscle, and hip adductor muscle activities using a surface electromyograph. We compared vaginal pressure for each measurement position (supine, standing, half sitting, and load-lifting) by twoway analysis of variance with the task performed (rest, PFM contraction), using position as a variable factor, and multiple comparison tests. Additionally, we compared the rate of increase in muscle activity during PFM contraction in each position.

Results: For task factors, vaginal pressure and all tested muscle activity were significantly higher during PFM contraction. For position factors, vaginal pressure and all tested muscle activity showed significantly higher values in the half sitting and load-lifting. The rate of increase in muscle activity of the IO was higher than that of the other muscles in the supine, standing, and the half sitting.

Conclusion: It was suggested that IO increased the activity as a co-contraction muscle with PFM in the half sitting, supine, and standing in comparison with other muscles.

Key Words: Half sitting position, Pelvic floor muscle, Internal oblique muscle, Co-contraction, Stress Urinary Incontinence 


\title{
Relationship between Health-related Quality of Life and Exercise Capacity or Physical Activity Status in Children and Adolescents with Congenital Heart Disease
}

\author{
Goro FUJITA, PT, Takayoshi NAKAMURA, PT \\ Department of Rehabilitation, The Jikei University Katsushika Medical Center \\ Goro FUJITA, PT, Aiko OTAKA, PT, MSc, Yasuhide NAKAYAMA, PT, PhD \\ Department of Rehabilitation, The Jikei University Hospital \\ Takashi URASHIMA, MD, PhD \\ Department of Pediatrics, The Jikei University School of Medicine \\ Kazushige KOBAYASHI, MD, PhD, Masahiro ABO, MD, PhD \\ Department of Rehabilitation, The Jikei University School of Medicine
}

Purpose: The purpose of this study was to evaluate the relationship between health-related quality of life (HRQOL) and exercise capacity or physical activity status in children and adolescents with congenital heart disease (CHD) and healthy controls.

Methods: The study included 22 patients with CHD (mean age $13 \pm 3$ years) and 22 healthy controls (13 \pm 3 years). We assessed HRQOL, exercise capacity (using cardiopulmonary exercise testing), physical activity level, and exercise habits, and evaluated the relationships between each index.

Results: Among the HRQOL subscores, physical well-being (PW) was significantly lower in children with CHD $(\mathrm{p}<0.05)$. In children with CHD, there was a significant correlation between $\mathrm{PW}$ and anaerobic threshold ( $\mathrm{rs}=0.472, \mathrm{p}<0.05$ ), but not between $\mathrm{PW}$ and peak oxygen uptake. Further, there was a correlation between physical activity level and PW in children with $\mathrm{CHD}(\mathrm{rs}=0.504, \mathrm{p}<0.05)$, and children with CHD who had regular exercise habits had higher PW $(\mathrm{p}<0.05)$ than those without regular exercise habits.

Conclusion: HRQOL in children with CHD is associated with anaerobic threshold and daily physical activity status.

Key Words: Congenital heart disease, Health-related quality of life, Exercise capacity, Physical activity, Cardiac rehabilitation 


\title{
Impaired Postural Stability in Patients with Adult Spinal Deformity: Evaluation Based on Computerized Stabilometry
}

\author{
Shinya SAKURAI, PT, Kumiko ARAMOTO, PT, Tetsuya OHARA, MD \\ Department of Rehabilitation, Meijo Hospital \\ Hironori TANABE, MD, DMSc, Tetsuya OHARA, MD \\ Department of Orthopaedic \& Spine Surgery, Meijo Hospital
}

Purpose: Recent insights suggest that spinal deformity impacts various functional disorders. However, there are few reports of examinations of postural balance in patients with adult spinal deformity (ASD), and the effect of kyphotic changes in ASD on postural balance remains unclear. The aim of this study was to evaluate standing balance and the influence of kyphotic changes on standing balance in patients with ASD.

Method: We investigated 46 female patients with ASD (age: $67.4 \pm 10.0$ years) and compared the results with those of 21 age-matched healthy female adults. Based on the sagittal vertical axis (SVA), patients were allocated to the SVA $>100 \mathrm{~mm}$ and SVA $<100 \mathrm{~mm}$ groups. Postural stability was examined using a stabilometer. Using the stabilometer, the sway of the gravity center was measured at the upright position with eyes open for 60 seconds. We used 2 parameters for evaluation: the enveloped area (ENV), which measures the degree of sway of the gravity center, and total track length (LNG), which measures the sway length in the standing posture.

Results: The mean ENV in the patient group was $5.3 \pm 3.9 \mathrm{~cm}^{2}$, whereas that in the control group was 3.3 $\pm 1.1 \mathrm{~cm}^{2}$, revealing significantly larger postural instability in the patient group compared to that in the control group. The LNG in the patient group was significantly worse than that in the control group. It was also shown that postural instability was significantly larger in the patients with SVA $>100 \mathrm{~mm}$ than that in patients with SVA $<100 \mathrm{~mm}$.

Conclusion: The results of the present study demonstrated impairments in postural stability in patients with ASD. In these patients, kyphotic changes were associated with greater postural instability. Therefore, exercises that delay the progression of kyphosis may be crucial for postural stability in patients with ASD.

Key Words: Adult spinal deformity, Postural stability, Stabilometry 


\title{
Longitudinal Examination of Factors Related to Shoulder Pain in High School Baseball Players
}

\author{
Yuichi JYUMONJI, PT \\ Sukagawa Hospital Rehabilitation Room \\ Yuichi JYUMONJI, PT, Eiki TSUSHIMA, PT, PhD \\ Hirosaki University Graduate School of Health Sciences \\ Hideo KOBAYASHI, MD, PhD, Kenya TSUDA, MD \\ Sukagawa Hospital Orthopedic Surgery
}

Background: Baseball players who have shoulder pain due to throwing have reduced shoulder joint range of motion and shoulder external rotation muscle strength, which are also recognized as general features of baseball players. The purpose of this study was to examine whether these factors affect the development of shoulder pain through prospective studies.

Methods: In members of high school baseball teams, shoulder joint function was evaluated by field position. during the off-season and observations were collected over a 2-month period during the season. Subsequently, evaluation items that affected the occurrence of shoulder pain were analyzed.

Result: Twenty-four out of the 84 participants developed shoulder pain, and multiple logistic regression analysis extracted the shoulder rotator strength ratio and field position as significant variables. Consideration: A decrease in the shoulder rotator strength ratio significantly affected the occurrence of shoulder pain in all field positions. Although stretching has been reported to be important for preventing throwing disorders, attention must also be paid to muscle strengthening exercises that take into account the balance of rotator muscle strength.

Key Words: Baseball, Throwing obstacles, Shoulder pain, Shoulder joint function, Shoulder rotator muscle strength 
The Course of Patient Reported Outcome and Physical Function after Surgery with LSS

Hiroto TAKENAKA, PT, MA, Kasuri NISHIHAMA, PT, MS, Junya SUZUKI, PT, MS, Atsuki ITO, PT

Department of Rehabilitation, Asahi Hospital

Hiroto TAKENAKA, PT, MA, Hideshi SUGIURA, MD, PhD

Department of Physical and Occupational Therapy, Nagoya University Graduate School of Medicine

Shuntaro HANAMURA, MD, PhD, Mitsuhiro KAMIYA, MD, PhD

Department of Orthopedic Surgery, Asahi Hospital

Purpose: The purpose of this study was to prospectively clarify the course of postoperative patientreported outcomes and physical function in patients with lumbar spinal stenosis.

Methods: We analyzed the data of 78 patients who completed pre-, 1-month, 3-month, 6-month, and postoperative evaluations (maximum follow-up was 12 months). Thirty-seven patients had undergone fusion surgery $(68.4 \pm 10.5$ years), and 41 patients had undergone decompression surgery (68.9 \pm 7.8 years). We evaluated the Japanese orthopaedic association back pain evaluation questionnaire (JOABPEQ), visual analog scale (VAS) scores (low back pain, lower extremity pain, and numbness), 6-minute walk test results, and trunk muscle strength. This study was approved by the institutional review board of the authors' affiliated institutions.

Results: In the fusion surgery and decompression surgery, the four scales of the JOABPEQ, VAS (low back pain, lower limb pain and numbness), and 6-minute walk distance improved from 1 month after surgery. On the other hand, lumbar dysfunction of the JOABPEQ improved from 6 months after surgery. Trunk muscle strength improved from 3 months after decompression surgery.

Conclusions: The course of postoperative JOABPEQ and physical function was clarified. These are considered to be useful as explanations for surgery and target values for the postoperative course.

Key Words: Lumbar spinal stenosis, Change of postoperative outcome, Patient-reported outcomes, Physical function 


\section{Factors Predicting Functional Outcome in Acute Stroke Patients: A Multicenter Study}

Koki HASEGAWA, PT

Department of Rehabilitation, Sainokuni Higashiomiya Medical Center

Yuji FUJINO, PT, PhD, Tadamitsu MATSUDA, PT, PhD

Department of Physical Therapy, Faculty of Health Science, Juntendo University

Kazuhiro FUKATA, PT, MS

Department of Rehabilitation, Saitama Medical University International Medical Center

Hiroshi MIKI, PT, MS

Department of Rehabilitation, Tokyo Saiseikai Central Hospital

Yohei KOBAYASHI, PT

Department of Rehabilitation, Saitama Sekishinkai Hospital

Hirofumi SATO, PT, MS

Department of Rehabilitation, Medical Technology Department, Saitama Citizens Medical Center

Purpose: We investigated potential predictors of functional outcome in acute stroke patients.

Methods: This multicentric prospective cohort study evaluated NIH stroke scale (NIHSS), Brunnstrom recovery stage, Trunk control test, Revised version of the ability for basic movement scale, Scale for contraversive pushing (SCP), Scale for the assessment and rating of ataxia, Functional ambulation category (FAC), and stroke type (cerebral infarction or hemorrhage) in 447 acute stroke (294 cerebral infarction) patients at the outset of their rehabilitation and assessed whether these measures predicated functional outcome (home discharge or rehabilitation hospital discharge). Multivariable logistic regression analysis was used to evaluate outcome predictors.

Results: The identified significant factors associated with outcome were NIHSS (OR: 1.234, 95\%CI 1.1101.372, p<0.01), SCP (OR: 6.270, 95\%CI 1.461-26.904, p<0.05), FAC (OR: 0.527, 95\%CI 0.417-0.668, p<0.01), and stroke type (OR: $3.369,95 \% \mathrm{CI} 1.896-5.986$, $\mathrm{p}<0.05)$, with the percentage of correct classifications was $81.6 \%$.

Conclusion: It was suggested that evaluation data at the early mobilization is useful to predict the functional outcome.

Key Words: Acute stroke, Functional outcome, Functional evaluation at first time, Multicenter study 


\section{Castration-Induced Pelvic Floor Muscle Specific Atrophy and the Effect of Voluntary Wheel-Running Exercise}

Kiyoshi YOSHIOKA, PT, MSc, Tomohiro MATSUMOTO, PT, PhD, Kodai NAKAMURA, PT, MSc, Yoshifumi TSUCHIYA, PhD, Daiki SEKO, MSc, Yasuo KITAJIMA, PhD, Yusuke ONO, PhD Department of Muscle Development and Regeneration, Division of Organogenesis, Institute of Molecular Embryology and Genetics, Kumamoto University

Kiyoshi YOSHIOKA, PT, MSc

Rehabilitation Center for all customers with Stroke and Cerearovascular diseases Kumamoto Center

Yuichiro SAWADA, MD, PhD

Department of Urology, Ehime University Graduate School of Medicine

Purpose: Pelvic floor muscles play an important role in excretion control and thus maintaining their function is crucial to quality of life. The aim of this study was to examine muscle atrophy in a mouse model of androgen deprivation and the effect of voluntary wheel-running exercise on pelvic floor muscles.

Methods: Castration and sham-operation were performed on 3-month-old male mice (C57BL/6J). Castrated mice were randomly housed in cages equipped with a running wheel. After 8 weeks from the operation, muscle samples were collected from limb and perineal (Limb muscles: tibialis anterior, gastrocnemius, extensor digitorum longus, plantaris and soleus. Perineal muscles: bulbospongiosus and levator ani). Myofibers were isolated from extensor digitorum longus and peri-urethral sphincter and the diameters were measured.

Results: Castration significantly decreased perineal muscles' mass and myofiber diameter of periurethral sphincter, whereas limb muscles were not affected. Voluntary wheel-running exercise cannot ameliorate castration-induced muscle atrophy in pelvic floor muscles.

Conclusion: Whole-body exercise cannot prevent pelvic floor muscle loss caused by androgen deprivation and thus it is suggested that target-muscle-specific training needs to be considered for the intervention.

Key Words: Men's Health, Androgen, Skeletal Muscle 


\title{
Correlation between the Psychological Aspects of Exercise about Post-discharge and Exercise Time after Discharge in Hospitalized Patients in the Recovery Ward
}

\author{
Hiroto FUKUMOTO, PT, MSc \\ Toyama Prefectural Rehabilitation Hospital \& Support Center for Children with Disabilities \\ Hiroki FUKUSHIMA, MSc \\ University of Toyama Faculty of Human Development
}

Purpose: This study investigated the correlation between questionnaire survey responses and exercise time after discharge from the recovery period in hospitalized patients.

Methods: This study recruited 100 patients in the recovery ward. In addition to the questionnaire survey, we requested records on exercise time. The questionnaire was based on existing models and psychology theories on exercise adherence. Exercise time was measured for one month from the discharge day by placing a sticker on a calendar as a diary of self-activity.

Results: Data from 100 questionnaires and 35 exercise times were obtained. Factor analysis of the questionnaire, converged to 25 items and four factors. To verify the reliability, the $\alpha$ coefficient was calculated for 25 items, resulting in an $\alpha$ of 0.895 . Correlation analysis with the average exercise time after discharge was confirmed as a significant weak correlation as verification of validity.

Conclusion: The results suggested a relationship between the psychological aspects of exercise after discharge from the convalescent ward and exercise time after discharge.

Key Words: Psychological aspects, Exercise adherence, Questionnaire, Recovery ward 
Interlimb Coordination in Patients Undergoing Total Knee Arthroplasty

Hideyuki WANAKA, PT, MSc

Kobe Rosai Hospital

Akira IWATA, PT, PhD, Toshimitsu OHMINE, PT, MSc, Saki YAMAMOTO, PT, MSc

Faculty of Comprehensive Rehabilitation, Osaka Prefecture University

Yuki SANO, PT, MSc, Hikari SOMATOMO, PT, Haruhiko YASUDA, PT, Takashi NISHII, MD, PhD

Osaka General Medical Center

Toshimitsu OHMINE, PT, MSc

Shimada Hospital

Purpose: In this study, we compared interlimb coordination in patients before and after total knee arthroplasty (TKA) using the phase coordination index (PCI). Additionally, we investigated its association with other variables at each time.

Methods: This study included 55 patients who underwent TKA (mean age $76.1 \pm 7.4$ years). We assessed and compared the preoperative PCI with the PCI measured on the day before discharge (approximately 17 days after TKA). Pearson's correlation and multiple regression analyses were used to determine factors affecting the PCI.

Results: We observed no significant difference between the pre- $(6.73 \% \pm 3.09 \%)$ and post-TKA $(6.94 \%$ $\pm 2.97 \%)$ PCI. The pre-TKA PCI was significantly correlated with knee extension strength on the nonoperative side and knee pain on the operative side, and knee extension strength on the nonoperative side was selected as an independent variable in this study. Postoperatively, a significant correlation was observed only between the PCI and patient age.

Conclusions: This study showed that pre- and post-TKA interlimb coordination was comparable; however, different factors were associated with the pre- or post-TKA PCI.

Key Words: Total knee arthroplasty, Interlimb coordination, Phase coordination index 
Factors Related to Lung Compliance in Patients with Permanent Ventilation Dependence

Shinichi ONOZAWA, PT, MS

Asanogawa General Hospital Rehabilitation Center

Kanazawa University, College of Medical, Pharmaceutical and Health Sciences, Doctoral Program

Fujiko SOMEYA, MD, PhD

Kanazawa University, College of Medical, Pharmaceutical and Health Sciences, School of Health Sciences

Eiji NURIYA, MD

Department of Anesthesia, Asanogawa General Hospital

Objective: We aimed to clarify factors related to lung compliance in patients with permanent ventilation dependence.

Methods: This was a cross-sectional study. We measured the static lung compliance (Cstat) and dynamic lung compliance (Cdyn) of 29 patients, and compared the values between those with and without atelectasis, pleural effusion, and spontaneous breathing. Correlation analyses were performed to examine the relationships between lung compliances and ventilation status, demographic parameters, and biochemical blood data.

Results: Cstat was significantly lower in patients with atelectasis than in those without. Cstat and Cdyn were significantly correlated with the body mass index (BMI), rapid shallow breathing index (RSBI), alveolar arterial oxygen partial pressure difference, and days of ventilator management. Cstat was also significantly correlated with age, C-reactive protein, and the number of pneumonia episodes.

Conclusion: The negative influence of BMI on Cstat and Cdyn was high, suggesting the need for nutritional management against obesity. This study also suggested that Cstat is affected by atelectasis, the number of pneumonia episodes, and inflammatory blood response. The decreases in Cstat and Cdyn may affect the ventilation efficiency reflected by RSBI. It is necessary to examine whether the improvements in atelectasis and pneumonia frequency affect Cstat and the ventilation efficiency index in the future.

Key Words: Permanent ventilation dependence, Lung compliance, Related factors 


\title{
Cost-effective Analysis of Exercise Programs Designed for Fall Prevention among Healthy Younger Old Community-dwelling Adults
}

\author{
Gohei KATO, PT, PhD \\ Department of Physical Therapy, School of Rehabilitation Tokyo Professional University of Health Sciences \\ Yosuke KURACHI, PT
}

Karada Kobo

Purpose: The aim of this study is to reveal the cost-effectiveness of exercise programs designed for fall prevention among healthy younger old community-dwelling adults in Japan.

Methods: The analysis was conducted on behalf of public insurers for health and long term care services. Quality-adjusted life years (QALY) and expenses for health services and long-term care services were described in terms of "effectiveness" and "cost," respectively. The assumed subjects were healthy community-dwelling females $(n=1,000)$ and males $(n=1,000)$ aged 65 years old. The incremental cost-effective ratio (ICER) of the program was analyzed and simulated using a 10-year cycle Markov model (base case). The threshold for assessing cost-effectiveness was set at less than 5 million Japanese yen/QALY.

Results: The ICER for the female group was 1,550,900 Japanese yen/QALY, and 2,277,086 Japanese yen/QALY for the male group.

Conclusion: An exercise program for fall prevention among healthy younger old community-dwelling adults could be cost-effective in Japan.

Key Words: Younger Old Community-Dwelling Adults, Fall Prevention, Cost-Effective Analysis, Markov Model, Exercise Program 


\title{
The Effect of Exercise Therapy and the Impact of Joint Injection in Shoulder Joint Disease Patients with Night Pain Undergoing Physical Therapy
}

\author{
Hajime ITO, PT, MS, Kiyonori YO, PT, PhD, Yoko MATSUDA, PT \\ Department of Rehabilitation Hamawaki Orthopaedic Clinic \\ Hajime ITO, PT, MS, Teruhiko KAWAKAMI, MD, PhD \\ Kibi International University Graduate School of Health Sciences
}

Yusuke MUROFUSHI, PT, PhD

Tohto University Makuhari Human Care Department

Objective: This study aims to demonstrate the usefulness of exercise therapy for night pain improvement in patients with shoulder joint disease undergoing physical treatment. We examined the treatment course by the presence, and effect of joint injection or absence of night pain at the initial evaluation.

Methods: The subjects were 72 patients with unilateral shoulder joint disease undergoing physical treatment. Patients were classified into two groups based on the presence and absence of night pain at the initial evaluation. Then, the subjects in the group with night pain were further classified into two groups according to whether or not joint injection was performed at the initial examination. We analyzed the difference in the treatment course, including range of motion (ROM), visual analogue scale (VAS), and Athens Insomnia Scale (AIS) at 1 and 3 months after the start of treatment.

Results: In both groups, courses showed interaction. ROM, VAS on movement, AIS high degree of improvement were obtained in the night pain group. VAS on movement, high degree of improvement were obtained for one month later at the initial evaluation in the joint injection group.

Conclusion: Exercise therapy for patients with shoulder joint disease undergoing physical treatment can improve ROM, VAS, and AIS, regardless of the presence of night pain. In addition, pain can be improved early by joint injection at the initial visit.

Key Words: Shoulder joint disease patients, Night pain, Joint injection 
Kinematic Analysis of Lifting by Individuals with Lower Back Pain and Fear of Movement

Ren FUJII, PT, MS

Department of Rehabilitation, Medical Corporation Tanakakai Musashigaoka Hospital

Ren FUJII, PT, MS, Yuki NISHI, PT, MS, Shu MORIOKA, PT, PhD

Department of Neurorehabilitation, Graduate school of Health Science, Kio University

Ryota IMAI, PT, PhD

School of Rehabilitation Osaka Kawasaki Rehabilitation University

Shinichiro TANAKA, MD

Department of Rehabilitation Medicine, Medical Corporation Tanakakai Musashigaoka Hospital

Gosuke SATO, PT, PhD, Shu MORIOKA, PT, PhD

Neurorehabilitation Research Center, Kio University

Purpose: The purpose of this study was to investigate the characteristics of impaired trunk movements during lifting in people with lower back pain (WLBP) with fear of movement.

Methods: Twenty-six WLBP and eighteen pain-free healthy workers (HW) were recruited. We calculated the peak angular velocity of trunk and motion time during lifting an object. WLBP were evenly divided into low-fear and high fear groups. We compared the angular velocity of trunk with three groups and examined the relationship between these factors and pain-related factors.

Results: Our kinematic analyses revealed significant differences in the extension phase and the peak angular velocity of trunk extension in the first trial among the three groups. The peak angular velocity of trunk extension in first trial was significant correlated with TSK.

Conclusion: It was shown that lifting in people with lower back pain with fear of movement was characterized by the movement of the trunk extension.

Key Words: Lower back pain, Lifting, Fear of movement, Medical care worker 


\title{
Gait Analysis in Healthy Elderly People with and without Walking Aids
}

\author{
Shomaru ITO, PT, MS \\ Graduate School of International University of Health and Welfare \\ International University of Health and Welfare Hospital \\ Tatsuya IGAWA, PT, PhD, Tsuyoshi HARA, PT, PhD \\ International University of Health and Welfare \\ Hitoshi MARUYAMA, PT, PhD \\ Graduate School of International University of Health and Welfare
}

Objective: The purpose of this study was to examine the joint kinematics and kinetics during walking with three types of walking aids in healthy elderly people.

Methods: Nineteen healthy elderly people participated in this study. A three-dimensional motion capture system and force plates were used to obtain kinetic and kinematic data while walking on a level surface. All subjects walked without any aid and with walkers, rollators, and rollators with forearm support. We measured the peak values of lower extremity joint angles, moments, and ground reaction forces in healthy elderly people.

Results: The peak values of knee adduction moment (KAM) while using the aids were smaller than without any aid. Furthermore, KAM was significantly lower when walking with rollators and rollators with forearm support compared to with walkers.

Conclusion: The use of rollators and rollators with forearm support can help to reduce KAM during level walking in healthy elderly people.

Key Words: Gait analysis, Joint moment, Walking aids, Elderly 


\section{Periodic Outpatient Self-training Guidance Improved Physical Function and the Instrumental Activity of Daily Living in a Breast Cancer Patient with Multiple Brain Metastases: A Case Report}

Masahiko KUSABA, PT, PhD, Jun SAWADA, OT, Kaho GOMA, PT, Keisuke OURA, PT, MSc, Toshihiko EBISU, MD, PhD

Department of Rehabilitation Medicine, Kansai Electric Power Hospital

Utae KATSUSHIMA, MD, Kazuhiro YANAGIHARA, MD, PhD

Department of Medical Oncology, Kansai Electric Power Hospital

Takeshi ITO, Ph, MSc

Department of Pharmacy, Kansai Electric Power Hospital

Noboru MAKABE, RD

Center for Metabolism and Clinical Nutrition, Kansai Electric Power Hospital

Masahiko KUSABA, PT, PhD, Utae KATSUSHIMA, MD, Keisuke OURA, PT, MSc,

Takeshi ITO, Ph, MSc, Noboru MAKABE, RD, Toshihiko EBISU, MD, PhD,

Kazuhiro YANAGIHARA, MD, PhD

Kansai Electric Power Medical Research Institute

Introduction: We observed the effectiveness of periodic outpatient rehabilitation on physical function and the Instrumental Activity of Daily Living (IADL) in a patient with advanced breast cancer.

Case report: A 52-year-old woman with multiple brain metastases due to breast cancer visited our hospital for chemotherapy for 5 years. Before rehabilitation, the Frenchay Activities Index (FAI) for the evaluation of IADL was 18 points and the Short Physical Performance Battery (SPPB) for the evaluation of physical function was 6 points (balance: 1 point, gait: 4 points, and sit to stand: 1 point). Rehabilitation involved muscle strengthening exercise, balance exercise, and self-exercise guidance for 13 weeks; once for 20 minutes, 9 times in total, in the outpatient chemotherapy unit. After rehabilitation, the patient improved, with an FAI of 23 points and an SPPB is 9 points (balance: 3 points, gait: 4 points, and sit to stand: 2 points).

Conclusion: This report may suggest that periodic outpatient rehabilitation improved physical function, resulting in improvement of IADL in a breast cancer patient with multiple brain metastases.

Key Words: Palliative chemotherapy, Outpatient, Rehabilitation, Breast cancer, Brain metastasis 


\title{
Availability of Plantar Sensory Threshold for Fall Risk Determination
}

\author{
Mitsuru SATO, PT, PhD, Tohru NAKABO, PT, PhD, Arinori KAMONO, PT, PhD \\ Showa University \\ Kazuhiko YAMASHITA, Eng, PhD \\ Ryotokuji University
}

Purpose: Decline of somatosensory function is associated with falls in the elderly. This study investigated the relationship between loss of plantar sensory threshold and fall risk in the elderly.

Methods: The sensory thresholds of plantar foot were measured in 110 people using day service facilities for the elderly with long-term care needs (33 men and 77 women; mean age, 80.7 years), using a new sensory testing device with high reproducibility. In addition, physical and cognitive functions including lower extremity strength and gait speed were measured. Fall history during the past 12 months was determined retrospectively, and each measurement variable was compared between the faller and non-faller groups. The relationship between plantar sensory threshold and fall history was examined by calculating the odds ratios of important variables determined by logistic regression analysis with the presence of a fall event as the objective variable.

Results: There were significant differences in the plantar sensory threshold, ankle dorsiflexion angle, and ratio of men to women between the fallers and non-fallers. The odds ratio adjusted by sex was significant in the plantar sensory threshold and the ankle dorsiflexion angle.

Conclusions: The plantar sensory threshold will be valuable information to explain falls in the elderly requiring long-term care. The results of this study suggested that assessment including the plantar sensory threshold in addition to conventional indices would be more effective for determining the risk of falls in elderly people who require long-term care.

Key Words: Plantar sensation, Falls, Elderly, Requiring long-term care, Risk factors 


\section{Psychometric Properties of Mini-Balance Evaluation Systems Test in Patients with Acute Stroke}

Ren TAKEDA, PT, Tatsuya IGARASHI, PT, MSc, Aya OKUMA, PT, Kazuya HOSHINO, PT

Numata Neurosurgery \& Heart Disease Hospital

Tomohiro KOYAMA, PT

Numata Clinic

Kazuhiro MIYATA, PT, PhD

Department of Physical Therapy, Ibaraki Prefectural University of Health Sciences

Objective: We examined the validity, reliability, responsiveness, and interpretability of the Mini-Balance Evaluation Systems Test (Mini-BESTest) in patients during the acute phase of a stroke.

Method: This study included 42 patients in the acute phase of a stroke. We examined the correlations between Mini-BESTest scores and those of other similar scales, the internal consistency of the MiniBESTest, the correlation between score changes when using the Mini-BESTest and when using the existing balance evaluation scale, and the discriminative ability of the Mini-BESTest in assessing walking independence.

Results: Mini-BESTest scores showed significant correlations $(\mathrm{r}=0.36-0.83)$ with balance scores and other similar measures, and good internal consistency $(\alpha=0.88)$. Score improvements when using the Mini-BESTest and when using the existing balance evaluation scale showed a significant correlation $(r=$ 0.84 ), but the discriminative ability of the Mini-BESTest in assessing walking independence was low.

Conclusion: We have suggested that the Mini-BESTest is a valid tool for assessing the balance ability of patients during the acute phase of a stroke. We have demonstrated the validity, reliability, responsiveness, and interpretability of the Mini-BESTest.

Key Words: Mini-BESTest, Stroke, Balance, Psychometric properties 


\section{The Characteristics of the Site-specific Body of Muscle Mass and the Physical Functions in Community-dwelling Elderly Men Under Long-term Care}

Jitsuhito FUKUO, PT, MSc

Department of Rehabilitation, Innoshima General Hospital

Satoshi MURAKI, PhD

Graduate School of Design, Kyushu University

Objective: The aim of this study was to investigate the characteristics of the physical functions and the site-specific muscle mass in elderly men under long-term care.

Methods: Fifty-three community-dwelling elderly ( $\geq 65$ years of age) men participated in this study. The participants were classified into a robust elderly group (group R) and an elderly under long-term care group (group C). Their physical functions were evaluated by questions 6-10 of the Kihon checklist subitems. Muscle thickness was evaluated in eight regions of the body using B-mode ultrasound, and body height, body weight, and body mass index (BMI) were measured.

Results: The total and individual physical function scores of group $\mathrm{C}$ were higher than those of group $\mathrm{R}$. The anterior and posterior lower legs were the only sites where the muscle thickness of group $\mathrm{C}$ was significantly lower than that in group $\mathrm{R}$.

Conclusion: The findings suggested that elderly men under long-term care often have the loss of muscle mass, particularly in the anterior and posterior lower legs.

Key Words: Community-dwelling elderly men under long-term care, Sarcopenia, Site-specific body of muscle mass, Physical functions 


\title{
Relationship between Blood Pressure Changes in the First Sitting Position and Neurological Deterioration in Patients with Acute Ischemic Stroke
}

\author{
Hiroki NAKASHIMA, PT, MSc, Taro TANIZAKI, PT, Mitsuhiro SERIZAWA, PT, \\ Shohei ITO, PT, MSc, Yuki MIYAZAKI, PT, Atsuko KANDA, PT \\ Department of Rehabilitation, Ichinomiya Municipal Hospital \\ Sayaka SOBUE, PT \\ Department of Rehabilitation, Toyota East Rehabilitation Hospital
}

Objective: To elucidate the relationship between blood pressure changes in early sitting position and neurological deterioration (ND) in patients with acute ischemic stroke.

Methods: A total of 165 patients hospitalized within $24 \mathrm{~h}$ after the onset of ischemic stroke were seated for $>10 \mathrm{~min}$ on bed at the earliest possible time. Blood pressure changes at 3 or $10 \mathrm{~min}$ after the sitting position were compared to those obtained in supine position. We divided the patients into two groups, with and without ND, indicating $\geq 2$ points based on National Institutes of Health Stroke Scale, which worsened within 7 days after admission. The association between variables included in the multivariate model and occurrence of ND was examined.

Results: A total of 25 patients (15.2\%) exhibited ND. On univariate analysis, perforator stroke, hemoglobin Alc level, high-density lipoprotein cholesterol (HDL-C) level, systolic blood pressure at 10 min while sitting, and elevated and reduced blood pressure while sitting exhibited a statistical significance $(\mathrm{p}<0.05)$. On multivariate analysis, perforator stroke (odds ratio [OR], 5.35; 95\%confidence interval [CI], 1.67-17.20), HDL-C level (OR, 0.96; 95\%CI, 0.92-0.99), and reduced blood pressure while sitting (OR, 14.1; 95\%CI, 3.93-55.70) demonstrated to be independent predictive factors.

Conclusion: Reduced blood pressure during an early sitting position shows to be related to ND within 7 days after admission in patients with acute ischemic stroke.

Key Words: Ischemic stroke, Neurological deterioration, Blood pressure change 


\title{
Descending Stairs in Patients after Total Knee Arthroplasty Fails to Reproduce Efferent Knee Joint Extension Moment in Healthy Volunteers
}

\author{
Taiki FURUMOTO, PT, Ayane KATAYAMA, PT, Yu MATSUI, PT, Yuka KAWAMURA, PT, \\ Ken TOMONARI, PT, Shinsuke KATOH, MD, PhD \\ Department of Rehabilitation, Tokushima University Hospital \\ Taiki FURUMOTO, PT, Daisuke HAMADA, MD, PhD, Koichi SAIRYO, MD, PhD \\ Department of Orthopedics, Institute of Biomedical Sciences Tokushima University Graduate School
}

Purpose: To clarify whether a patient can reproduce the elongation moment of the efferent knee joint in patient of the same age while descending stairs after total knee arthroplasty (TKA).

Methods: The subjects were 8 patients in the TKA group who were able to perform step-down movements in one step and 1 step 1 year after undergoing TKA, and 10 healthy volunteers of the same age. In the descending motion analysis, lower limb muscle activity was measured using a threedimensional motion analyzer and ground reaction force meter by measuring the joint angle, joint moment, joint power, and surface electromyogram of the sagittal plane. All measured data were compared for the main measurement item, first peak of knee extension moment (20\%), and second peak of knee extension moment (80\%).

Results: In the TKA group, the elongating moment of the efferent knee joint during the lowering movement was significantly lower in both the early stance phase and late stance phase than that in the healthy group.

Conclusion: One year after the TKA, the efferent knee joint extension moment of healthy individuals of the same age cannot be reproduced while descending stairs.

Key Words: Total knee arthroplasty, Descending stairs, Efferent knee joint extension moment 


\section{Effects and Safety of Early Resistance Training in Hospitalized Older Patients with Heart Failure: A Randomized Controlled Trial}

Masami INOKUMA, PT, MSc, Yoshihisa NAMASU, OT, MSc, Akinari FUKUSHI, PT, MSc, Yuhei YAMASHITA, OT, MSc, Takuya KUWAHARA, PT, MSc, Harue NAKANO, PT, Tatsunori SHITARA, PT, Mayumi TAKAYANAGI, OT, Hiroko KAZAMA, PT

Division of Rehabilitation Medicine, Gunma Prefectural Cardiovascular Center

Masami INOKUMA, PT, MSc, Shigeru USUDA, PT, PhD

Gunma University Graduate School of Health Sciences

Chikako YOSHIDA, Nrs

Nursing Department, Gunma Prefectural Cardiovascular Center

Taisuke NAKADE, MD, Makoto MURATA, MD, Hitoshi ADACHI, MD, Shigeru OSHIMA, MD

Division of Cardiology, Gunma Prefectural Cardiovascular Center

Purpose: The present study aimed to determine the safety and feasibility of early RT and its effects on physical function among hospitalized elderly patients with heart failure.

Methods: The remaining patients were randomly assigned to either a resistance training (RT) or a control group. The primary outcome was knee extension muscle strength, and the secondary outcome was comfortable walking speed and the SPPB.

Results: The pathological status of only one patient worsened. Knee extension muscle strength and comfortable walking speed significantly interacted, and the effect size of resistance training on knee extension muscle strength and comfortable walking speed was moderate, and small on the SPPB in the RT group.

Conclusions: These results suggest that early resistance training can be safe and effective for hospitalized elderly patients with heart failure when indications, contraindications, and exclusions are appropriately applied and training is gradually increased in stages.

Key Words: Hospitalization period, Older patients with heart failure, Resistance training, Safety, Effects 
Effect of Inspiratory Muscle Training on Physical Activity in Patients with Chronic Obstructive Pulmonary Disease: A Multicenter, Randomized, Placebo-controlled Trial

Kazuki OKURA, PT, MSc

Division of Rehabilitation Medicine, Akita University Hospital

Hitomi TAKAHASHI, PT, PhD

Department of Physical Therapy, School of Health Sciences, International University of Health and Welfare

Takanobu SHIOYA, MD, PhD

Geriatric Health Facility Nikoniko-en

Yuki IIDA, PT, PhD

Department of Physical Therapy, School of Health Sciences, Toyohashi Sozo University

Takeshi INAGAKI, PT, PhD

Division of Rehabilitation Medicine, Chiba University Hospital

Tomoya OGAWA, PT, MSc

Department of Rehabilitation, Tosei Genaral Hospital

Tomoko OKUJO, PT

Department of Rehabilitation, Sakaide City Hospital

Hiromasa TSUTSUI, PT

Department of Rehabilitation, Konan Hospital

Eri HISANO, PT

Rehabilitation Center, Kanamecho Hospital

Shinjiro MIYAZAKI, PT, MSc

Rehabilitation Center, KKR Takamatsu Hospital

Objective: This study aimed to investigate the efficacy of inspiratory muscle training (IMT) on physical activity in patients with chronic obstructive pulmonary disease (COPD).

Methods: Fifty-nine outpatients with stable COPD were enrolled in this study. The participants were randomly assigned to either an IMT group (set intensity at $>30 \%$ of their maximal inspiratory pressure $\left.\left[\mathrm{PI}_{\text {max }}\right]\right)$ or a sham training group $\left(<10 \% \mathrm{PI}_{\max }\right)$. Patients of both groups underwent 30 breath IMT sessions twice daily for 3 months. The $\mathrm{PI}_{\max }, 6$-minute walk distance (6MWD), daily step counts, and moderate-to-vigorous intensity physical activity time (MVPA) were recorded before and after the 3 months intervention.

Results: Fifty participants (23 from the IMT group and 27 from the sham training group) completed the entire assessment. Significant interactions in the $\mathrm{PI}_{\max }$, daily step counts, and MVPA were observed. Only patients in the IMT group showed significant improvement in these attributes after the intervention. The 6MWD significantly improved in patients of both groups, and no significant interaction was observed.

Conclusion: This study suggests that the IMT improved the $\mathrm{PI}_{\max }$ and could improve physical activity in patients with stable COPD.

Key Words: Chronic obstructive pulmonary disease, Inspiratory muscle training, Physical activity, Exercise capacity 


\title{
Characteristics of the Five Functional Domains about Gait Performance: Comparison between 3-10 Years Old Children and Healthy Adults
}

\author{
Hiroki MANI, PT, PhD, Tadayoshi ASAKA, PT, PhD \\ Department of Rehabilitation Science, Faculty of Health Sciences, Hokkaido University \\ Saori MIYAGISHIMA, PT, PhD, Yui SATO, PT, MS \\ Division of Rehabilitation, Sapporo Medical University Hospital, Sapporo Medical University \\ Naoki KOZUKA, PT, PhD \\ Department of Physical Therapy, School of Health Sciences, Sapporo Medical University \\ Kenji TANEDA, PT, MS, Takahiro INOUE, PT, MS \\ Graduate School of Health Sciences, Hokkaido University \\ Kenta TAKEDA, PT, PhD \\ Department of Rehabilitation for the Movement Functions, Research Institute of National Center for \\ Persons with Disabilities
}

Purpose: This study aimed to characterize the five functional domains (pace, variability, rhythm, asymmetry, and postural control) of gait performance in 3-10-year-old children.

Methods: A total of 76 healthy children aged 3-10 years and 14 young adults were included in this study. The child population was divided into four age groups: 3-4, 5-6, 7-8, and 9-10 years. Participants were instructed to walk at self-selected speed barefoot on a 6-m walkway. Spatiotemporal gait parameters (step length (SL), step velocity (SV), step time (ST), stance time (STT), and swing time (SWGT)) were calculated using a 10-camera VICON 3D motion analysis system. In addition, mean values, standard deviation, coefficient variation (CV), and symmetry index (SI) were calculated. These gait parameters were divided into five domains: pace (SL, SV, and SWGT_CV), variability (SL_CV, SV_CV, ST_CV, and STT_CV), rhythm (ST, STT, and SWGT), asymmetry (ST_SI, STT_SI, and SWGT_SI), and postural control (SW, SW_CV, and SL_SI).

Results: Differences in spatiotemporal parameters as regards a gait pattern were not significant in children aged 7-10 years and in adults (pace, rhythm, and asymmetry domains), whereas group differences in variability and postural control domains were significant in all children and adult groups. Conclusion: Development of each of the five functional domains with respect to gait performance varies, and gait variability and stability mature longer than the gait pattern.

Key Words: Pediatric, Motor development, Gait 


\section{Characteristics of Lower Muscle Flexibility and Generalized Joint Laxity at Around Age of Peak Height Velocity in Adolescent Male Soccer Players}

Ryota KURATSUBO, PT, JSPO-AT, Ryota MUNAKATA, PT, MSc

Department of Rehabilitation, Kitasato Institute Hospital, Kitasato University

Ryota KURATSUBO, PT, JSPO-AT, Yuji TAKASAWA, MD, PhD

Department of Sports Medicine, Juntendo University Graduate School of Medicine

Hiroyuki WATANABE, PT, JSPO-AT, PhD

Department of Rehabilitation, School of Allied Science, Kitasato University

Hiroyoshi MASUMA, PT, MSc

Department of Rehabilitation, Kitasato University Hospital

Masumi YOSHIMOTO, PT, MSc

Department of Physical Therapy, Faculty of Health and Medical Science, Teikyo Heisei University

Objective: Decreased lower muscle flexibility and increased joint laxity, which are affected by bone growth, are considered intrinsic risk factors of sports injuries in adolescent players. However, little is known about changes in lower muscle flexibility and joint laxity at around age of peak height velocity (APHV).

Purpose: To examine the characteristics of lower muscle flexibility and joint laxity at around APHV in male soccer players.

Methods: Thirty-three adolescent soccer players participated in this cross-sectional study. Eligibility criteria included a) availability of height data from 4th grade of elementary school to 1st grade of junior high school and b) no pain throughout the body. Muscle flexibility of the hamstrings, quadriceps, and gastrocnemius was measured. Joint laxity was measured using the general joint laxity test. APHV was calculated based on the date height was measured. Maturity status was defined as the difference between actual age and APHV. Based on maturity status, participants were divided into three groups (G1: actual age $\leq 6$ months before APHV, G2: actual age $\leq 6$ months after APHV, G3: actual age 6-12 months after APHV)

Results: Flexibility of the gastrocnemius in the G1 group $\left(-1.7 \pm 4.3^{\circ}\right)$ was significantly lower than in the G2 group ( $\left.3.8 \pm 5.3^{\circ}\right)$, and the G1 group had significantly lower joint laxity $(1.8 \pm 1.0$ points) than the G3 group (3.3 \pm 1.3 points).

Conclusion: Our results suggest that muscle flexibility of the gastrocnemius and joint laxity before APHV were lower than those after APHV.

Key Words: Adolescent male soccer players, Age of peak height velocity, Maturational index, Lower muscle flexibility, Generalized joint laxity 


\title{
Sports Exercise can Induce Cortical Expansion of Upper Limb in Individuals with Complete Spinal Cord Injury
}

\author{
Tomoya NAKANISHI, PT, MSc, Hirohumi KOBAYASHI, PhD, Kimitaka NAKAZAWA, PhD \\ Department of Life Science, Graduate School of Arts and Science, The University of Tokyo \\ Tomoya NAKANISHI, PT, MSc \\ Japan Society for the Promotion of Science \\ Kento NAKAGAWA, PhD \\ Faculty of Sport Sciences, Waseda University \\ Hirohumi KOBAYASHI, PhD \\ Shinshu Sports and Medical College
}

Objective: Functional magnetic resonance imaging (fMRI) was used to clarify upper limb representation in the primary motor cortex (M1) in individuals with complete spinal cord injuries who had a history of playing sports.

Methods: Finger muscle and upper arm muscle contraction tasks during fMRI were conducted for seven individuals with complete spinal cord injury (SCI) and six healthy subjects, and the amount of brain activation was quantified. In addition, the brain regions that correlated with the number of years spent playing sports were calculated using population analysis.

Results: M1 activation size during finger muscle contraction was greater in the SCI group than in the healthy group. Further, brain activation during upper arm muscle contraction was correlated with the number of years spent playing sports in the population analysis.

Conclusion: After spinal cord injury, representation of the finger in the primary motor cortex was expanded by injury-induced plasticity, whereas representation of the upper arm was expanded by using dependent plasticity. This result suggests that intense physical activity such as sports is neurologically recommended in rehabilitation after SCI.

Key Words: Spinal cord injury, Primary motor cortex, Brain reorganization, Sports, fMRI 


\section{Use of Patient-based Outcomes Obtained from the Simple Shoulder Test Evaluation to Predict Treatment Strategies for Japanese Patients with a Rotator Cuff Tear}

Junichi KAWAKAMI, PT, MS, Masaki KARASUYAMA, PT, MS

Department of Physical Therapy, School of Rehabilitation, Kyushu Nutrition Welfare University

Junichi KAWAKAMI, PT, MS, Takashi NAGAMATSU, PT, MS, Takaki IMAI, PT, PhD,

Nobuya HARADA, PT, Yu KUDOU, PT

Kurume University School of Medicine Graduate School

Masafumi GOTOH, MD, PhD

Department of Orthopedic Surgery, Kurume University Medical Center

Koumei MATSUURA, MD

Department of Orthopedic Surgery, Saiseikai Yahata General Hospital

Aya YORITANI, PT

Department of Rehabilitation, Kugimiya Orthopedic \& Rehabilitation Clinic

Kazuya MADOKORO, PT, PhD

Department of Physical Therapy, Technical School of Medical and Welfare Ryokuseikan

Naoto SHIBA, MD, PhD

Department of Orthopedic Surgery, Kurume University

Purpose: We used a patient-based outcomes evaluation (Simple Shoulder Test) to predict treatment strategies for Japanese patients with a rotator cuff tear.

Methods: Subjects were 229 patients with a rotator cuff tear who provided answers to 12 questions in the Japanese version of the Simple Shoulder Test. Decision tree and propensity score analyses were used to calculate odds ratios.

Results: Patient were grouped according to their responses. Surgical group included patients who had night pain, pain at rest, limited physical mobility, and were unable to work; Conservative therapy group, those who had no night pain, no pain at rest, and were mobile; and Intermediate group those who provided mixed responses to these questions. The odds of patients selecting surgical treatment was 11.50 greater in Surgical group compared to Conservative therapy group and 3.47 greater in Group Surgical group compared to Intermediate group.

Conclusion: Four questions about night pain, pain at rest, mobility, and ability to work were predictive in the patient's selection of treatment and possibly the need for targeted physical therapy from an early stage.

Key Words: Shoulder Joint, Rotator Cuff Tear, Decision tree analysis, Patient-Based Outcomes 


\title{
Examination of Factors Related to Death Outcome of Patients Admitted to Psychiatric Ward with Pneumonia
}

\author{
Takahiro KANDA, PT, Hiromi FUKUDA, PT, Yusuke ISHIBASHI, PT, MSc, Muneyoshi NISHIDA, PT \\ Department of Rehabilitation, Akitsu Kounoike Hospital \\ Yoshihito TSUBOUCHI, OT, MSc \\ Department of Rehabilitation, Faculty of Health Sciences, Nara Gakuen University \\ Motoasa KOU, MD \\ Division of Psychiatry, Akitsu Kounoike Hospital
}

Objective: This study aimed to elucidate the factors associated with the outcome of death in patients who received physical therapy (PT) due to pneumonia in our hospital ward.

Method: This study included subjects who were admitted to our psychiatric ward, had nursing and healthcare-associated pneumonia (NHCAP), and underwent physical therapy between January 2015 and November 2018. The subjects were divided into two groups (survival group and death group) based on the outcome at 120 days from PT initiation. Cox proportional hazards analysis was used to examine the factors related to the outcome of death.

Result: The analysis included 81 people, of which 31 (38.3\%) died within 120 days of PT initiation. Cox proportional hazards analysis revealed that age and onset body mass index (BMI) were significantly associated with the outcome of death.

Conclusion: The results suggested that the outcome of death in patients admitted to the psychiatric ward with NHCAP was influenced by age and onset BMI.

Key Words: Psychiatric ward, Death outcome, Nursing and Healthcare associated Pneumonia 